\title{
Multicriteria Decision Aid Applications to Support Risk Decisions in the Marine Environment: Locating Suitable Transshipment Areas
}

\author{
Dimitrios I. Stavrou, Nikolaos P. Ventikos \\ National Technical University of Athens, School of Naval Architecture and Marine Engineering, \\ Laboratory for Maritime Transport (Maritime Risk Group), 9 IroonPolytechniou str. Zografou 15773, Greece, \\ E-mail:dstaurou@mail.ntua.gr,niven@deslab.ntua.gr
}

Received 13 February 2017

Accepted 26 February 2017

\begin{abstract}
The aim of this paper is to develop an MCDA model to support decision makers in the marine environment. The UTASTAR method is employed for the selection of the best area for ship-to-ship transfer of cargo. The method relies on the hypothesis that both the actions and the corresponding criteria interact with each other over time, constructing and formulizing the decision aid model. The use of real data for the tuning of the model parameters may lead to the optimal compatibility between model and decision-maker cognition.
\end{abstract}

Keywords: Multicriteria decision aid models, ship-to-ship transfer of cargo, aggregation-disaggregation methods, global criterion model.

\section{Introduction}

Shipping is a risky business as well as a risky operation due to different issues involving both operational (Ballis and Stathopoulos, 2003) and trading factors (Roumboutsos et al. 2004); the operational factors are related to the complex and frequently hostile sea environment, whereas the trading factors refer to the primary target of the shipping companies, which is to maximize their profits in a highly competitive and demanding market (Sambracos and Ramfou, 2001). An interesting and very promising way to deal with decision support problems in the marine environment comes from the employment of different multicriteria decision aid (MCDA) methodologies.

In most cases, MCDA methods are preference modelling approaches that are based on binary relations of the alternatives under the outranking relation law or ordinal regression approaches, which employ methodologies based on additive value models (Lopez et al. 2008). The modelling of the decision makers' (DMs') preferences in outranking relations is achieved by the direct interrogation of the DM and the analyst, in which the DM must determine the weights of the evaluation criteria, as well as the preference, indifference, and veto thresholds (Zopounidis, 2001).

There are several representative works on this approach (Roy, 1985; Roy and Bouyssou, 1993). A plethora of related examples can be found in the work of Siskos and Tsotsolas (2015).

On the other hand, the additive value models refer to the order of preference of a set of actions or alternatives followed by a consistent family of criteria (Greco et al., 2008, 2011; Hurson and Siskos, 2014). There exist many different approaches when referring to the additive value functions. For example, multiattribute utility theory (MAUT) is based on the construction of a global utility function that corresponds to the DM preferences (Keeney and Raiffa, 1976; Farquhar, 1984; Fishburn, 1967; Figueira et al. 2005). In 
MAUT theory, a direct interrogation process is employed to elicit information from the DM concerning the trade-offs among the conflicting criteria, attributes, or points of view with the objective of constructing the global preference model in the form of a utility function that the DM implicitly uses to make decisions (Zopounidis, 2001). Thus, MAUT offers a consistent and analytical evaluation of the alternative actions with regard to the corresponding criteria.

Alternatively, additive value functions can be built by employing the global criterion method (Siskos, 2008). This method is based on the aggregationdisaggregation or, in other words, analytical approach that refers to the analysis (disaggregation) of the global preferences (judgement policy) of the DM to identify the criteria aggregation model that underlies the preference result (Doumpos and Zopounidis, 2001) for the development of one or more additive value functions. Thus, the analytical approach theory relies on the assumption that the knowledge of the mechanism that DMs have in mind can be extracted and used to rank different alternatives that are governed by the same principles or rules. The process is applied to a predefined set of actions or alternative solutions $A_{R}$. At first, an initial order of preference integrating both real and imaginary alternatives is built. The ultimate purpose is to construct a model that consists of the marginal functions of the selected criteria by applying special LP techniques. The ranking of the actions obtained through these functions on $A_{R}$ should as consistent as possible with the initial ranking of actions (Lakiotaki et al. 2009). Finally, the extracted model is extrapolated to the rest of the alternative set of actions (Spyridakos and Yannacopoulos, 2015).

The main representative of the analytical approach comes from the UTA (Utility Additives) family, which was initially proposed by Jacquet-Lagreze and Siskos $(1982,2001)$. The primary goal of the UTA methods is the inductive inference of one or more additive value functions using a preference order of reference actions. The UTA methods belong to the wide family of analytic global criterion methods with monotonic regression. This paper focuses on the implementation of the UTASTAR method, which was initially proposed by Siskos and Yannacopoulos (1985) as an improved version of the original UTA method.

In the original UTA method, each alternative action comes with a unitary defined error (Grigoroudis et al. 2004), which corresponds to the correction of the value of each criterion in order, the additive value function, to verify the order of preference of the reference actions. The UTA method finally calculates the marginal value function of each criterion as a result of the solving a linear problem (LP) with the objective function, the error function, and the constraints that arise from the nature of the problem. The UTASTAR method aims to remedy the weakness of the original UTA method by minimizing, through the error function, the total dispersion of the points that corresponds to the value of each criterion with regard to the reference actions. More specifically, the UTASTAR introduces the meaning of the double error function $\left(\sigma^{+}\left(a_{k}\right)\right.$ and $\left.\sigma^{-}\left(a_{k}\right)\right)$, taking into account situations of both underestimation and overestimation of the marginal value function (Matsatsinis, 2010).

To examine the effectiveness of the UTASTAR method for decision models from the marine sector, the method is applied to the problem of choosing a suitable area for ship-to-ship (STS) transfer of cargo.

Thus, the aim of this paper is to develop an MCDA model based on the analytical global criterion method with monotonic regression for the location of the most suitable STS transfer area. To achieve this goal, the rest of the paper is organized as follows: First, the current context of the MCDA additive value functions is presented. Next, in Section 2, the link between the analytical approaches and the UTASTAR method is properly described, and in Section 3, the method is applied for the selection of the most appropriate STS transfer location. In Section 4, the results are presented and discussed. Finally, Section 5 concludes the paper.

\section{From the analytical approach to the UTASTAR methodology}

When dealing with multi-attribute decision problems by applying Roy's (1985) general methodological framework, the majority of the developed decision models are based on the axiom that the final decision is the result of the evaluation of the different actions or alternatives followed by a consistent family of criteria. Hence, there is a type of inductive inference process in which the general conditions of the problem determine the final decision. This "cause and effect" approach was reconsidered by Jacquet-Lagreze and Siskos (1982), who stated that the final decision can be the result of knowledge of the cognitive mechanism by which the DMs decide instead of an inductive inference process. 
Thus, the analyst can model the DMs' way of thinking and apply this knowledge to make decisions regarding other alternatives from the same set of actions. This philosophy is the fundamental principle of aggregationdisaggregation or simply analytical methods where the decision and criteria interact with each other over time and co-construct the decision model (Siskos, 2008). According to the theory of analytical methods, the first step is to collect the data relevant to the decision problem and then to determine the actions and the corresponding criteria. Next, a model is constructed based on the DM's order of preference for the predefined actions. Finally, the constructed model is compared with the order of preference of actions and, for absolute relevance, the constructed model is extrapolated to the rest of the actions; otherwise the model is properly corrected to fit the initial order of preference.

The most representative family of analytical methods is the UTA family. A flowchart of the analytical approach is shown in Figure 1.

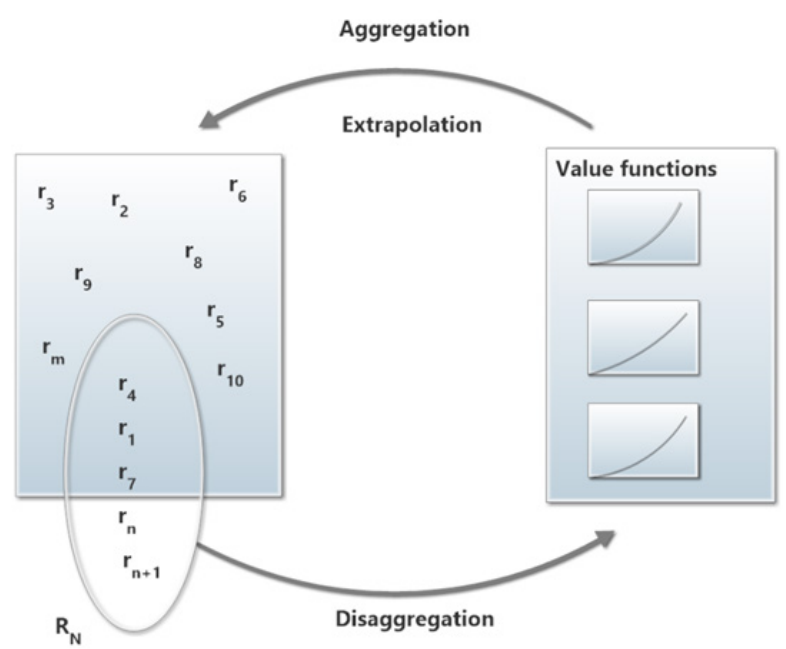

Fig. 1. The concept of the analytical approach through the UTA methodologies includes a given order of preference of the selected actions which is used as a mean to determine the corresponding value functions during the disaggregation process. Next comes the aggregation process where the extracted model is extrapolated to the rest of the alternative set of actions.

In UTA methods, the analyst takes for granted the preferences of the DM regarding a given set of reference actions $A_{R}$. The preference actions could be either real, taken from the original set of actions, or imaginary, which means that they are built on the selected criteria.

Given the order of preference of the selected actions, the UTA method applies special techniques of linear programming to determine the corresponding value functions, through which the criteria are aggregated into a global criterion per action. That is why this kind of modelling is also known as the "global criterion model".

The value function $u$ of a given set of actions $A=$ $\left\{a_{1}, a_{2}, \ldots, a_{m}\right\}$, followed by a consistent family of criteria $g_{1}, g_{2}, \ldots, g_{n}$, is expressed by the relationship:

$$
\begin{gathered}
u(\boldsymbol{g})=u\left(g_{1}, g_{2}, \ldots, g_{n}\right) \\
\boldsymbol{g}(a) \rightarrow u[\boldsymbol{g}(a)]
\end{gathered}
$$

where $g_{i^{*}}$ and $g_{i}^{*}$, correspond to the worst and best values of the criterion $g_{i}$, and $u[\boldsymbol{g}(a)]$ is a real number that is known as the value of action $\alpha$.

Thus, the global value of each action can be expressed by an additive value function of the following form:

$$
u(\boldsymbol{g})=\sum_{i=1}^{n} u_{i}\left(g_{i}\right)
$$

Under the normalization constraints,

$$
\begin{gathered}
\sum_{i=1}^{n} u_{i}\left(g_{i}^{*}\right)=1 \\
u_{i}\left(g_{i *}\right)=0, \forall i=1,2, \ldots
\end{gathered}
$$

where $u_{i}, i=1,2, \ldots n$ represent monotonic non-descent functions $g_{i}$ known as marginal value functions.

In order to implement a UTA model, two conditions should be in force: preference independence among the selected criteria and the property of consistency or monotony, which means that:

when $u[\boldsymbol{g}(a)]>u[\boldsymbol{g}(b)]$, action $\alpha$ is preferred to $\mathrm{b}(6)$ when $u[\boldsymbol{g}(a)]=u[\boldsymbol{g}(b)]$, DM is indifferent between action $\alpha$ and $b$

The UTASTAR is an improved version of the original UTA method (Jacquet-Lagreze and Siskos, 1982). It refers to the evaluation of a set of actions $A=\left\{a_{1}, a_{2}, \ldots, a_{n}\right\}$ according to a consistent family of criteria $g_{i}=\left\{g_{1}, g_{2}, \ldots, g_{m}\right\}$ based on an order of preference of actions given by the DM. During the model implementation, an additive value function is constructed from the selected criteria with the objective of being consistent with the initial order of preference. The improvement of the UTASTAR method comes from the introduction of the meaning of double error to deal with underestimations (which is also dealt by the original UTA method) as well as overestimations of the 
marginal functions. Another innovative characteristic of the UTASTAR is the transformation of the partial values of each criterion scale of the reference actions through equidistant spaces $w_{i j}$ to facilitate the resolution of the LP (Siskos, 2008). A detailed description of the UTASTAR method can be found in the work of Siskos (2008) or Matsatsinis (2010). In brief, the UTASTAR consists of the following four successive steps:

Step 1: The actions and the corresponding criteria are determined. Discretized criterion scales are made and the global value of the actions $u\left[\boldsymbol{g}\left(a_{k}\right)\right], k=1,2, \ldots, m$ is initially expressed through the use of the marginal value functions $u_{i}\left(g_{i}\right)$. Next, the transformation $w_{i j}$ of each value function is conducted according to the relationships:

$$
\begin{gathered}
w_{i j}=u_{i}\left(g_{i}^{j+1}\right)-u_{i}\left(g_{i}^{j}\right) \geq 0, \\
\forall i=1,2 \ldots n \text { and } \xi=1,2, \ldots \alpha_{\iota}-1 \\
u_{i}\left(g_{i}^{1}\right)=0, \forall i=1,2 \ldots n \\
u_{i}\left(g_{i}^{j}\right)=\sum_{t=1}^{j-1} w_{i t}, \\
\forall i=1,2, \ldots n \text { and } \xi=2,3, \ldots \alpha_{i}-1
\end{gathered}
$$

Step 2: The error functions $\sigma^{+}$and $\sigma^{-}$of the underestimation and overestimation of the preference actions accordingly are integrated to each value function and the differences between the pairs (first, second), (second, third), and so on of the order of preference are written accordingly:

$$
\begin{array}{r}
\Delta\left(\alpha_{k}, a_{k+1}\right)=u\left[g\left(a_{k}\right)\right]-\sigma^{+}\left(a_{k}\right)+\sigma^{-}\left(a_{k}\right)- \\
{\left[u\left[g\left(a_{k+1}\right)\right]-\sigma^{+}\left(a_{k+1}\right)+\sigma^{-}\left(a_{k+1}\right)\right](11)}
\end{array}
$$

Step 3: The following LP is solved:

$$
[\min ] z=\sum_{k=1}^{m}\left[u\left[\sigma^{+}\left(a_{k}\right)+\sigma^{-}\left(a_{k}\right)\right]\right.
$$

Under the constraints:

$$
\begin{aligned}
\Delta\left(\alpha_{k}, a_{k+1}\right) \geq \delta, \text { when } \alpha_{k}>a_{k+1} \\
\Delta\left(\alpha_{k}, a_{k+1}\right)=0, \text { when } \alpha_{k} \sim a_{k+1}
\end{aligned}
$$

$$
\begin{aligned}
& \sum_{i=1}^{n} \sum_{j=1}^{a_{i}-1} w_{i j}=1 \\
& w_{i j} \geq 0, \sigma^{+}\left(a_{k}\right) \geq 0 \\
& \sigma^{-}\left(a_{k}\right) \geq 0, \forall i, j \text { and } k
\end{aligned}
$$

Step 4: The existence of multi-optimal or semi-optimal solutions of the relative LP is checked through the calculation of the weighted average of the additive value functions that maximize the following objective functions. When a lack of robustness occurs, an additional constraint is applied to the existing ones of the previous hyper-polyhedron, which is:

$$
\sum_{a \in A_{R}}\left[\sigma^{+}(a)+\sigma^{-}(a)\right] \leq z^{*}+\varepsilon
$$

where $Z^{*}$ is the optimal error value of step 3 , and $\varepsilon$ corresponds to a very small positive number (or zero).

The new linear program should give $n$ new solutions that are able to optimize, in the new hyperpolyhedron, the linear functions:

$$
u_{i}\left(g_{i}^{*}\right)=\sum_{j=1}^{a_{i}-1} w_{i j}, \forall i=1,2 \ldots n
$$

If the stability of the model is adequate, the final decision corresponds to the mean utility function of $n$ meta-optimal solutions.

\section{Use of UTASTAR to choose STS transfer area}

According to the problem, a large oil cargo is to be delivered from the Persian Gulf to a final destination somewhere in north Europe (point B of Fig. 2). For both operational and financial reasons, the stakeholders have decided that the oil will be carried in parcels by Suezmax tankers from the Persian Gulf (point A of Fig. 2) through the Suez Canal with the objective of delivering each parcel to a Very Large Crude Carrier (VLCC) tanker by conducting an STS transfer operation. As the stakeholders now have to decide on the most suitable STS transfer area, there are four alternative locations under consideration: Cyprus $(\mathrm{Cr})$, Crete $(\mathrm{K})$, Malta $(\mathrm{M})$, and Gibraltar $(\mathrm{G})$. Information regarding the special characteristics of a sea area 
suitable for STS transfer operations can be found in IMO (2010) and OCMF (2013).

After careful examination of the alternative locations, which is beyond the scope of this paper, four different categories of criteria have been chosen to evaluate each STS transfer area: operational, economic, environmental, and safety/security criteria.

Operational criterion: This criterion refers to the operational potentialities that each location can offer to

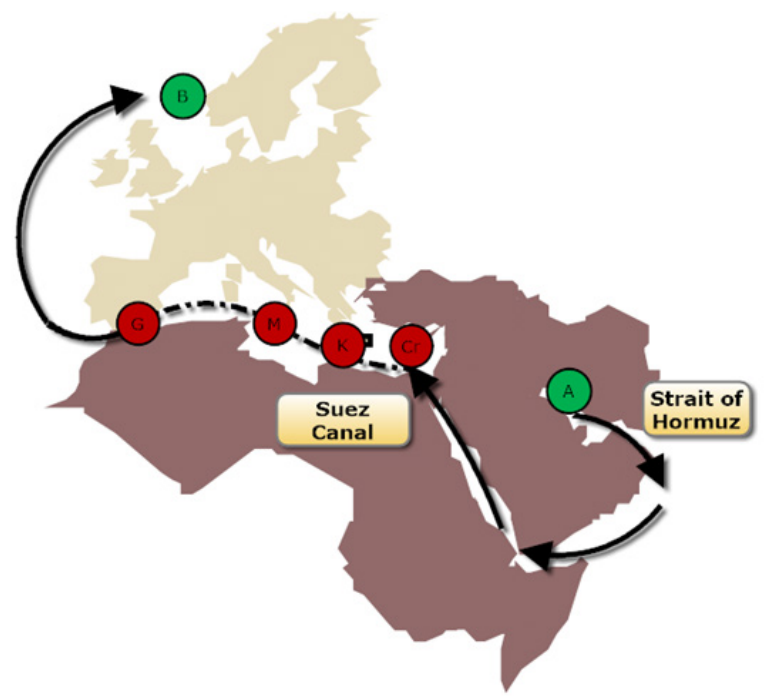

Fig. 2. Problem definition of the STS transfer area.

the transfer operation. For example, it includes the different ways (stationary or under way) in which the transfer operation can be conducted, the familiarization of the local authorities with the facilitation of the STS transfer process, the relative experience of the STS transfer provider who is responsible for providing the person in overall advisory control (POAC), the relevant STS transfer equipment, and finally the proximity of the facilities to provide critical equipment in case of emergency, such as oil pollution. The operational criterion takes values from zero, which refers to the worst operational conditions, where no sub-criterion is satisfied, to four, which refers to the best option, where all sub-criteria are satisfied. Finally, this is a positive qualitative criterion, which means that the larger the value of the criterion, the higher the value of the action for the stakeholder.
Economic criterion: This consists of the subcriteria that are relevant to the expenses that the stakeholder should cover to conduct the STS transfer operation in each suggested area. For example, it refers to the cost of the fees the stakeholder must pay to the authorities, the total cost of fuels and personnel for the transition to the STS transfer area, the payment for the STS provider services, and other additional operational costs. In order to evaluate the economic criteria, it is assumed that each economic sub-criterion makes a weighted contribution to the total cost of the STS transfer. The economic criterion is a quantitative criterion and is negative, which means that the lower the value, the more suitable is the location for the transfer and vice versa.

Environmental criterion: This is related to the prevailing weather or sea conditions that may affect the success of the operation, including the existence of sufficient room in which to conduct the transfer (especially transfers carried out under way), the traffic density of the proposed transfer area (too high a density may lead to early transfer suspension), the proximity of sensitive or protected areas, and finally the presence of a sheltered environment able to protect vessels from the forces of nature. This criterion is a qualitative criterion and is given by an empirical equation explained in detail in the work of Stavrou et al. (2016). Finally, the criterion has a negative meaning, which means that the lower the value, the more suitable is the location for the transfer. To evaluate the environmental criterion, it is assumed that each environmental sub-criterion makes a weighted contribution to the total cost of the STS transfer.

Safety and security criterion: This criterion refers to the geo-political status of the proposed transfer area, including the potential for unpredictable situations due to unstable geo-political relations between nearby nations. This criterion also accounts for the possibility of terrorist activity that may endanger crew, vessel, and cargo safety. A further factor is historical data regarding incidents or accidents within the area under consideration. It is a qualitative criterion and has a positive meaning, which means that the higher the value, the more suitable is the location for the transfer.

The summarized data for each location according to the selected criteria are shown in Table 1.

Table 1. Multi-criteria evaluation of the proposed STS locations

\begin{tabular}{|c|c|c|c|c|}
\hline Criterion & $\begin{array}{c}\text { Operational } \\
(0-4)\end{array}$ & $\begin{array}{c}\text { Environmental } \\
(0-100)\end{array}$ & $\begin{array}{c}\text { Economic } \\
\left(\$ \times 10^{3}\right)\end{array}$ & $\begin{array}{c}\text { Safety/Security } \\
(0-2)\end{array}$ \\
\hline Sense & Positive & Negative & Negative & Positive \\
\hline Cyprus & 2 & 44 & 31.7 & 1 \\
\hline Crete & 0 & 48 & 25.3 & 2 \\
\hline Malta & 4 & 59 & 15.9 & 2 \\
\hline Gibraltar & 3 & 38 & 19.2 & 2 \\
\hline
\end{tabular}


After a short discussion with the stakeholder, the analyst proceeds with the construction of four virtual actions or reference profiles. For example, regarding the question of choosing by instinct one of the four different alternatives, the stakeholder chooses Gibraltar due to its better environmental profile. The second choice of the stakeholder is Malta due to its low cost, and so on. The order of preference according to the stakeholder's cognition is shown in Table 2, while Table 3 depicts the corresponding discretized criterion scales.
Next, the transformation of Equation (1) is applied:

$$
\begin{aligned}
& u[g(A)]=w_{11}+0.5 w_{12}+w_{21}+w_{22}+w_{23}+0.5 w_{24} \\
& +w_{31}+w_{32}+0.17 w_{33}+w_{41}+w_{42} \\
& u[g(B)]=w_{11}+w_{12}+w_{31}+w_{32}+0.67 w_{33}+w_{41} \\
& +w_{42} \\
& u[g(\Gamma)]=w_{11}+w_{21}+w_{22}+0.5 w_{23}+w_{31} \\
& +0.17 w_{32}+w_{41} \\
& u[g(\Delta)]=w_{21}+0.83 w_{22}+w_{41}+w_{42}
\end{aligned}
$$

Table 2: Adapted table for the UTASTAR implementation.

\begin{tabular}{lccccc}
\hline Criterion & Operational & Environmental & Economic & $\begin{array}{c}\text { Safety/ } \\
\text { Security } \\
(0-2)(+)\end{array}$ & Order of Preference \\
Scale & $(0-4)(+)$ & $(0-100)(-)$ & $\left(\$ \times 10^{3}\right)(-)$ & $(0-2)$ & 1 \\
\hline A & 3 & 38 & 19 & 2 & $\mathbf{2}$ \\
B & 4 & 59 & 16 & 1 & $\mathbf{3}$ \\
C & 2 & 44 & 25 & 2 & 4 \\
D & 0 & 48 & 32 & & \\
\hline
\end{tabular}

Table 3: Discretized criterion scales

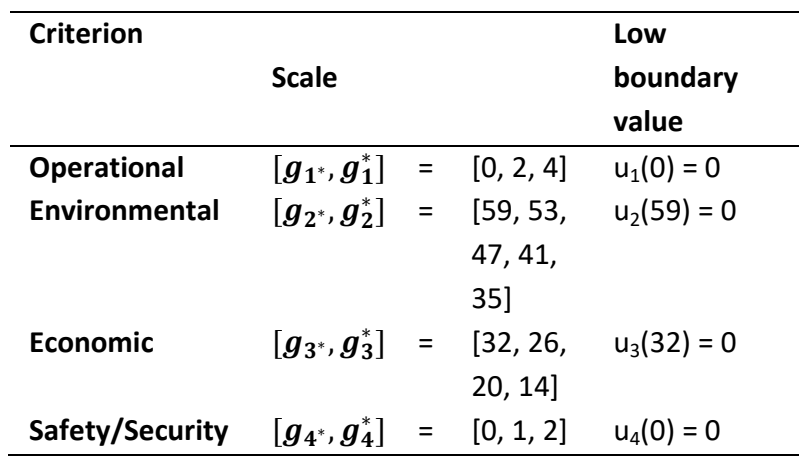

From the implementation of the first step of the algorithm, the value function of each action is calculated, applying the linear regression technique whenever needed. To do so, the following boundary conditions are also taken into account: $u_{1}(0)=u_{2}(59)=u_{3}(32)=u_{4}(0)=0$.

Thus, the value function of each action is given by the following equations:

$$
\begin{aligned}
& u[g(A)]=0.5 u_{1}(4)+0.5 u_{1}(2)+0.5 u_{2}(41)+0.5 u_{2}(35) \\
& +0.83 u_{3}(20)+0.17 u_{3}(14)+u_{4}(2) \\
& u[g(B)]=u_{1}(4)+0.33 u_{3}(20)+0.67 u_{3}(14)+u_{4}(2) \\
& u[g(\Gamma)]=u_{1}(2)+0.5 u_{2}(47)+0.5 u_{2}(41)+0.83 u_{3}(26) \\
& +0.17 u_{3}(20)+u_{4}(1) \\
& u[g(\Delta)]=u_{1}(0)+u_{2}(48)+u_{3}(32)+u_{4}(2)
\end{aligned}
$$

According to the second step, the differences between the corresponding pairs are calculated according to the order of preference of the actions:

$$
\begin{aligned}
& \Delta(A, B)=0.5 w_{12}-0.67 w_{21}-0.67 w_{22}-w_{23} \\
& -0.5 w_{24}-0.33 w_{31}-0.33 w_{32} \\
& +0.5 w_{33}-\sigma_{A}^{+}+\sigma_{A}^{+}+\sigma_{B}^{+}-\sigma_{B}^{+} \\
& \Delta(B, \Gamma)=w_{11}+0.5 w_{12}+0.17 w_{22}+w_{23}+0.5 w_{24} \\
& +w_{31}+w_{32}+0.17 w_{33}-\sigma_{B}^{+}+\sigma_{B}^{+} \\
& +\sigma_{\Gamma}^{+}-\sigma_{\Gamma}^{+} \\
& \Delta(\Gamma, \Delta)=-w_{11}-0.17 w_{22}-0.5 w_{23}-w_{31}-w_{32} \\
& +w_{42}-\sigma_{\Gamma}^{+}+\sigma_{\Gamma}^{+}+\sigma_{\Delta}^{+}-\sigma_{\Delta}^{+}
\end{aligned}
$$

In the third step, the LP is solved, taking into account a threshold value of $\delta=0.01$. The last row of the corresponding table shows the optimal value of the relative weights.

The solution of the LP gave a feasible solution but not a unique one. For that reason, it is necessary to carry out a robustness analysis in order to determine the corresponding solutions that verify the problem. To achieve this goal, the objective function is transformed per Equation (17). According to the results, $\mathrm{z}=0$, and thus the robustness analysis refers to those solutions that maximize the weight of each criterion $(\varepsilon=0)$. As all errors have zero values, they can be omitted and thus a new LP arises where the four objective functions are shown. 
Table 4: Table of the LP.

\begin{tabular}{|c|c|c|c|c|c|c|c|c|c|c|c|c|c|c|c|c|c|c|c|c|}
\hline$w_{11}$ & $w_{12}$ & $w_{21}$ & $w_{22}$ & $w_{23}$ & $w_{24}$ & $w_{31}$ & $w_{32}$ & $w_{33}$ & $w_{41}$ & $w_{42}$ & & & & $\overline{\mathrm{rs} \sigma}$ & $\overline{\text { and }}$ & & & & & \\
\hline 0 & -0.5 & 0 & 0 & 0.5 & 0.5 & 0 & 0 & 0.5 & 0 & 0 & -1 & 1 & 1 & -1 & 0 & 0 & 0 & 0 & $\geq$ & 0.01 \\
\hline 0 & 0.5 & 0 & 0 & -0.5 & -0.67 & 0 & 0.83 & 0.17 & 0 & 0 & 0 & 0 & -1 & 1 & 1 & -1 & 0 & 0 & $\geq$ & 0.01 \\
\hline 1 & 0 & 1 & 1 & 1 & 0.67 & 1 & 0.17 & 0 & 0 & 1 & 0 & 0 & 0 & 0 & -1 & 1 & 1 & -1 & $\geq$ & 0.01 \\
\hline 0 & -0.5 & 0 & 0 & 0.5 & 0.5 & 0 & 0 & 0.5 & 0 & 0 & 0 & 0 & 0 & 0 & 0 & 0 & 0 & 0 & $=$ & 1 \\
\hline 0.09 & 0.353 & 0.109 & 0.08 & 0.01 & 0.11 & 0 & 0 & 0.12 & 0.132 & 0 & 0 & 0 & 0 & 0 & 0 & 0 & 0 & 0 & & $z$ \\
\hline
\end{tabular}

Table 5. LP of meta-optimization for the problem of the STS transfer area location.

\begin{tabular}{ccccccccccccc}
\hline $\boldsymbol{w}_{\mathbf{1 1}}$ & $\boldsymbol{w}_{\mathbf{1 2}}$ & $\boldsymbol{w}_{\mathbf{2 1}}$ & $\boldsymbol{w}_{\mathbf{2 2}}$ & $\boldsymbol{w}_{\mathbf{2 3}}$ & $\boldsymbol{w}_{\mathbf{2 4}}$ & $\boldsymbol{w}_{\mathbf{3 1}}$ & $\boldsymbol{w}_{\mathbf{3 2}}$ & $\boldsymbol{w}_{\mathbf{3 3}}$ & $\boldsymbol{w}_{\mathbf{4 1}}$ & $\boldsymbol{w}_{\mathbf{4 2}}$ & & \\
\hline 0 & -0.5 & 0 & 0 & 0.5 & 0.5 & 0 & 0 & 0.5 & 0 & 0 & $\geq$ & 0.01 \\
0 & 0.5 & 0 & 0 & -0.5 & -0.67 & 0 & 0.83 & 0.17 & 0 & 0 & $\geq$ & 0.01 \\
1 & 0 & 1 & 1 & 1 & 0.67 & 1 & 0.17 & 0 & 0 & 1 & $\geq$ & 0.01 \\
\hline 1 & 1 & 1 & 1 & 1 & 1 & 1 & 1 & 1 & 1 & 1 & $=$ & 1 \\
\hline 1 & 1 & 0 & 0 & 0 & 0 & 0 & 0 & 0 & 0 & 0 & {$[\max ]$} & $u_{1}\left(g_{1}^{*}\right)$ \\
0 & 0 & 1 & 1 & 1 & 1 & 0 & 0 & 0 & 0 & 0 & {$[\max ]$} & $u_{2}\left(g_{2}^{*}\right)$ \\
0 & 0 & 0 & 0 & 0 & 0 & 1 & 1 & 1 & 0 & 0 & {$[\max ]$} & $u_{3}\left(g_{3}^{*}\right)$ \\
0 & 0 & 0 & 0 & 0 & 0 & 0 & 0 & 0 & 1 & 1 & {$[\max ]$} & $u_{4}\left(g_{4}^{*}\right)$ \\
\hline
\end{tabular}

After solving the meta-optimization LP, the following solutions are extracted:

Table 6: Robustness analysis and the final solution of the meta-optimization problem.

\begin{tabular}{ccccccccccccc}
\hline & & $\boldsymbol{w}_{\mathbf{1 1}}$ & $\boldsymbol{w}_{\mathbf{1 2}}$ & $\boldsymbol{w}_{\mathbf{2 1}}$ & $\boldsymbol{w}_{\mathbf{2 2}}$ & $\boldsymbol{w}_{\mathbf{2 3}}$ & $\boldsymbol{w}_{\mathbf{2 4}}$ & $\boldsymbol{w}_{\mathbf{3 1}}$ & $\boldsymbol{w}_{\mathbf{3 2}}$ & $\boldsymbol{w}_{\mathbf{3 3}}$ & $\boldsymbol{w}_{\mathbf{4 1}}$ & $\boldsymbol{w}_{\mathbf{4 2}}$ \\
\hline$[\max ]$ & $\boldsymbol{u}_{\mathbf{1}}\left(\boldsymbol{g}_{\mathbf{1}}^{*}\right)$ & 0.96 & 0.02 & 0 & 0 & 0.02 & 0 & 0 & 0 & 0 & 0 & 0 \\
{$[\max ]$} & $\boldsymbol{u}_{\mathbf{2}}\left(\boldsymbol{g}_{2}^{*}\right)$ & 0 & 0.02 & 0 & 0 & 0.02 & 0.96 & 0 & 0 & 0 & 0 & 0 \\
{$[\max ]$} & $\boldsymbol{u}_{\mathbf{3}}\left(\boldsymbol{g}_{\mathbf{3}}^{*}\right)$ & 0 & 0 & 0 & 0 & 0.01 & 0 & 0.31 & 0.68167 & 0 & 0 & 0 \\
{$[\max ]$} & $\boldsymbol{u}_{\mathbf{4}}\left(\boldsymbol{g}_{\mathbf{4}}^{*}\right)$ & 0 & 0 & 0 & 0 & 0.01 & 0 & 0.02 & 0 & 0 & 0.96 & 0.02 \\
\hline $\begin{array}{c}\text { Meta-optimal } \\
\text { solution }\end{array}$ & $\mathbf{0 . 2 4}$ & $\mathbf{0 . 0 1}$ & $\mathbf{0}$ & $\mathbf{0}$ & $\mathbf{0 . 0 1 5}$ & $\mathbf{0 . 2 4}$ & $\mathbf{0 . 0 8}$ & $\mathbf{0 . 1 7 0 4 2}$ & $\mathbf{0}$ & $\mathbf{0 . 2 4}$ & $\mathbf{0}$ \\
\hline
\end{tabular}

\section{Results and discussion}

The final solution of the LP corresponds to the marginal functions of Table 7, while the functions are depicted in Figure 3. The grey lines correspond to the initial solution of the LP, while the black line shows the final solution after the meta-optimization process. According to the results, the order of preference of the shareholder is verified:

$$
\begin{aligned}
& u[\boldsymbol{g}(A)]=0.875, \\
& u[\boldsymbol{g}(B)]=0.745, \\
& u[\boldsymbol{g}(\Gamma)] \\
& u[\boldsymbol{g}(\Delta)]
\end{aligned}
$$

As the model verifies the initial preference order, it can be further extrapolated to the evaluation of the rest of the selected actions. For example, in the case of Malta, the global value function will be:

$$
\begin{gathered}
u[\boldsymbol{g}(\text { Malta })]=u_{1}(4)+u_{2}(59)+u_{3}(15.9)+u_{4}(2) \\
=0.75
\end{gathered}
$$

Thus, the final ranking is as shown in Table 8.

In this paper, the order of preference of the reference actions emerged as a result of the interaction of the DM with the analyst. From the DM-analyst interaction, four fictitious actions emerged and were used to determine the corresponding marginal value functions. The results verified the initial order of preference and the value functions were used for the ranking of the rest of the actions. The interaction between DM and analyst was an 
Table 7: Marginal value functions for the problem of the STS transfer area location

\begin{tabular}{|c|c|c|c|}
\hline \multicolumn{2}{|r|}{ Criteria } & \multirow{2}{*}{ Feasible solution } & \multirow{2}{*}{ Meta-optimized solution } \\
\hline & Operational & & \\
\hline$u_{1}(0)$ & 0 & 0 & $\mathbf{0}$ \\
\hline$u_{1}(2)$ & $w_{11}$ & 0.09 & 0.24 \\
\hline \multirow[t]{2}{*}{$u_{1}(4)$} & $w_{11}+w_{12}$ & 0.443 & 0.25 \\
\hline & Environmental & & \\
\hline$u_{2}(59)$ & 0 & 0 & $\mathbf{0}$ \\
\hline$u_{2}(53)$ & $w_{21}$ & 0.1094 & $\mathbf{0}$ \\
\hline$u_{2}(47)$ & $w_{21}+w_{22}$ & 0.1894 & $\mathbf{0}$ \\
\hline$u_{2}(41)$ & $w_{21}+w_{22}+w_{23}$ & 0.2594 & 0.015 \\
\hline \multirow[t]{2}{*}{$u_{2}(35)$} & $w_{21}+w_{22}+w_{23}+w_{24}$ & 0.3724 & 0.255 \\
\hline & Economic & & \\
\hline$u_{3}(32)$ & 0 & 0 & $\mathbf{0}$ \\
\hline$u_{3}(26)$ & $w_{31}$ & 0 & 0.08 \\
\hline$u_{3}(20)$ & $w_{31}+w_{32}$ & 0 & 0.25 \\
\hline \multirow[t]{2}{*}{$u_{3}(14)$} & $w_{31}+w_{32}+w_{33}$ & 0.12 & 0.25 \\
\hline & Safety and security & & \\
\hline$u_{4}(0)$ & 0 & 0 & $\mathbf{0}$ \\
\hline$u_{4}(1)$ & $w_{41}$ & 0.1317 & 0.24 \\
\hline$u_{4}(2)$ & $w_{41}+w_{42}$ & 0.1317 & 0.24 \\
\hline
\end{tabular}

essential part that determined the nature of the obtained results.

Table 8: Final ranking of the corresponding alternatives for the selection of the STS transfer area.

\begin{tabular}{lll}
\hline Ranking & Candidate area & Value \\
\hline 1 & Gibraltar & 0.8800 \\
2 & Malta & 0.7500 \\
3 & Cyprus & 0.5015 \\
4 & Crete & 0.3498 \\
\hline
\end{tabular}

The implementation of the UTASTAR method demonstrates that the method has the capability to deal adequately with criteria of qualitative origins. This capability is very important especially when dealing with problems from the marine sector, where the need to apply qualitative scales in combination with qualitative ones is often unavoidable.

Another important observation comes from the step of the meta-optimization process. During the initial determination of the marginal value functions, the LP gave a feasible solution. This solution satisfies the problem constraints but is not the only one that does so. Thus, it was necessary to perform a robustness analysis in order to check the stability of the model through the determination of other solutions that satisfy the problem.

Thus, the robustness analysis through the metaoptimization process gives the ability to control the stability of the model, and the solutions of the LP constraints are the most representative ones. Moreover, the use of the weighted average for the selection of the optimal solution during the meta-optimization process confines the modelling of the DM's behavior within narrow limits. The weighted average solution corresponds to a risk-neutral DM and excludes cases of DMs who have low- or high-risk attitude.

Last but not least, it is essential to mention that an important advantage of the UTASTAR method is the ability to overcome problems of incomparability that can be met during the implementation of methods that are governed by the outranking relation law when a binary comparison between the actions is conducted. This is due to the fact that the final result is based on the aggregation of the marginal value functions of the selected criteria for each action rather than a binary comparison of the alternative actions. The incomparability remains a significant problem especially in the case of the employment of the ELECTRE methods. 

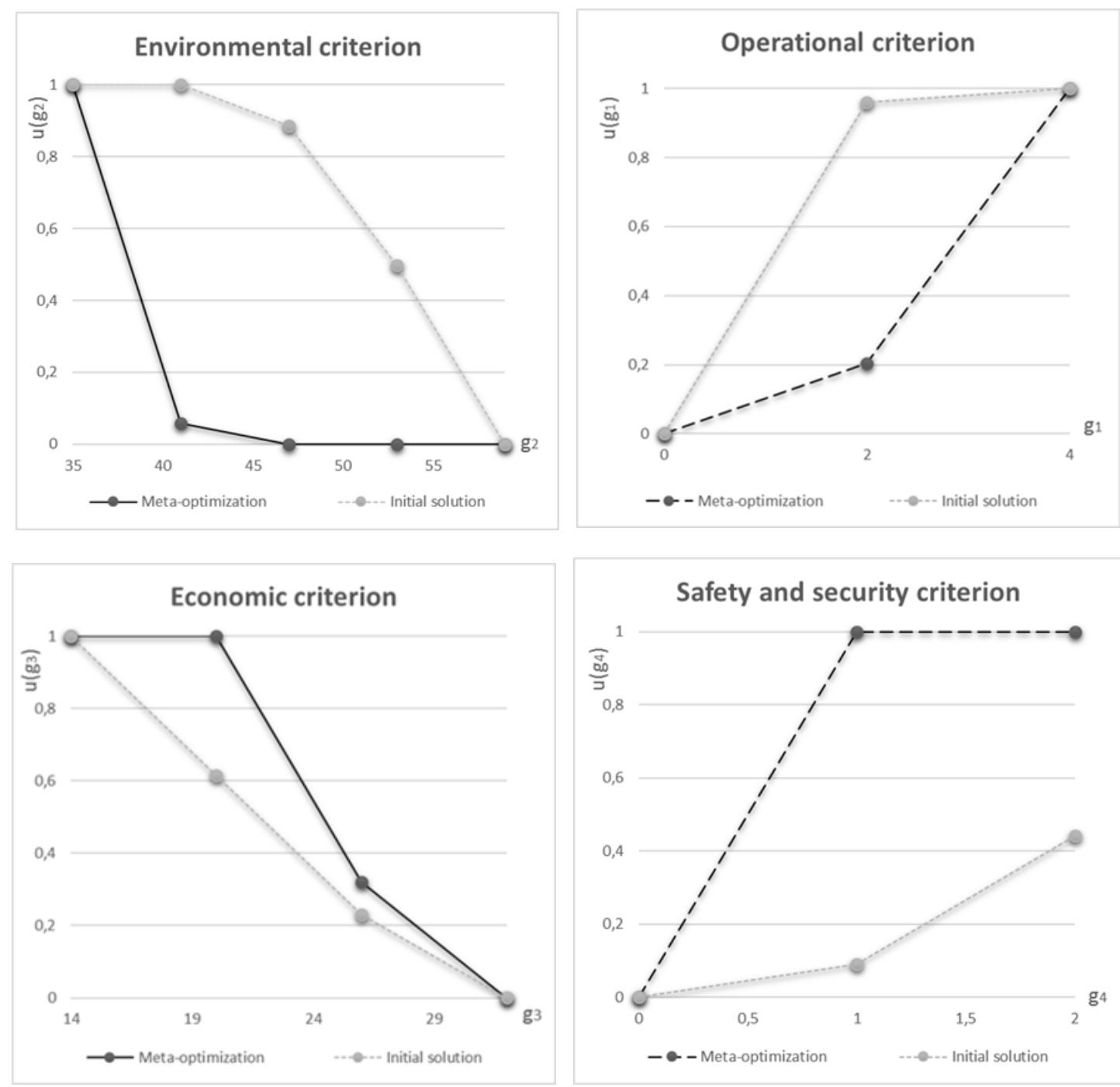

Fig. 3. Normalized additive value functions for the problem of the STS transfer area location.

\section{Conclusions}

The implementation of multi-attribute methodologies for decision problems in the marine environment is a very promising way to overcome problems of conflicting criteria in order to find a reliable and effective solution after the evaluation of the alternative actions. Often the comparison of two or more actions becomes very difficult due to the complex and frequently hostile sea environment. The unavoidable possibility of human error in combination with the unpredictable behaviour of the natural elements may compromise the success of a marine operation, causing adverse effects on humans and the environment as well as property loss. Thus, there is a pressing need to make the right decisions that will support operators when dealing with the various risks. On the other hand, the primary goal of the shipping companies always remains the maximization of profit together with the minimization of the use of necessary resources. The above two pillars can be adequately combined in the MCDA models, giving the shipping industry a new perspective as a result of the optimization of the evaluated alternatives. 


\section{References}

1. Ballis A, Stathopoulos A (2003) Criteria for Selecting Innovative Technologies for Maritime Transhipment Facilities, Oper Res Int J Vol.3 No.3 (2003): 213-227

2. Doumpos M, Zopounidis C (2001) Business failure prediction: A comparison of classification methods, Oper Res 2:303.

3. Farquhar P H, (1984) Utility assessment methods. Management Science 30(11) 1283-1300

4. Figueira J, Greco S, Ehrgott M (2005) Multiple Criteria Decision Analysis. State of the Art Surveys, Springer.

5. Fishburn P C (1967) Methods for estimating additive utilities. Management Science 13(7): 435-453

6. Greco S, Mousseau V, Słowiński R (2008) Ordinal regression revisited: Multiple criteria ranking using a set of additive value functions. Eur J Oper Res 191(2): 416-436

7. Greco S, Kadziński M, Mousseau V, Słowiński R (2011) ELECTREGKMS: Robust ordinal regression for outranking methods. Eur J Oper Res 214(10): 118135

8. Hurson C, Siskos Y, (2014) A synergy of multicriteria techniques to assess additive value models. Eur J Oper Res 238: 540-551

9. IMO (2010) Manual on Oil Pollution, Section I, Prevention of pollution. IMO publication

10. Jacquet-Lagreze E, Siskos J (1982) Assessing a set of additive utility functions for multicriteria decisionmaking: The UTA method. Eur J Oper Res 10: 151-164

11. Jacquet-Lagreze E, Siskos J (2001) Preference disaggregation: 20 years of MCDA experience. Eur J Oper Res 130: 233-245

12. Juan Carlos Leyva Lo'pez, Lizbeth Dautt Sa'nchez, Miguel Angel Aguilera Contreras (2008) A multicriteria decision support system with an evolutionary algorithm for deriving final ranking from a fuzzy outranking relation, Oper Res Int J (2008) 8:47-62, DOI 10.1007/s12351-008-0006-6

13. Keeney R L, Raiffa H (1976) Decisions with multiple objectives: Preferences and value trade-offs. John Wiley and Sons
14. Lakiotaki K, Delias P, Sakkalis V, Matsatsinis N F (2009) User profiling based on multi-criteria analysis: the role of utility functions, Oper Res Vol 9 (1): 3-16

15. OCMF (2013) Ship to Ship transfer guide for petroleum, chemical and liquefied cases, First edition, Scotland, UK

16. Roy B (1985) Methodologie Multicritere d'Aide a la Decision, Economica Paris

17. Roy B, Bouyssou D (1993) Aide multicritere a la decision: Methodes et cas. Paris: Economics

18. Roumboutsos A, Litinas N, Tobaloglou E, (2004) Management by Projects in Maritime Organizations, Oper Res Int J. Vol.4, No.3 (2004): 399-409

19. Sambracos, Ramfou I, (2001) Shipment Tracking Technologies and Customer Satisfaction, Oper Res Int J Vol 1 No I (2001): 43-54

20. Siskos E, Tsotsolas N (2015) Elicitation of criteria importance weights through the Simos method: A robustness concern. Eur J Oper Res (in press)

21. Spyridakos A, Yannacopoulos D (2015) Incorporating collective functions to multicriteria disaggregationaggregation approaches for small group decision making, Annals of Operations Research, April 2015 Vol 227(1):119-136, DOI 10.1007/s10479-014-1609-3

22. Stavrou D, Ventikos N, Siskos Y, (2016) Locating Ship-to-Ship (STS) transfer operations via MultiCriteria Decision Analysis (MCDA): A case study, Real Case Studies of Multiple Criteria Decision Making C. Zopounidis (ed) Springer (In press)

23. Zopounidis C, (2001) Preference disaggregation in financial modeling: Basic features and some examples, Oper Res, 1:263:263-283

24. Grigoroudis E, Doumbos M Zopounidis N, Matsatsinis $\mathrm{N}$ (Ed) 2004 Multicriteria decision analysis: Methodological approaches and applications, New Technologies, Athens

25. Matsatsinis N (2010) Decision Support Systems, New Technologies Athens 2004

26. Siskos I (2008) Decision Models 1st Edition Athens ISBN: 978-960-6759-10-9 